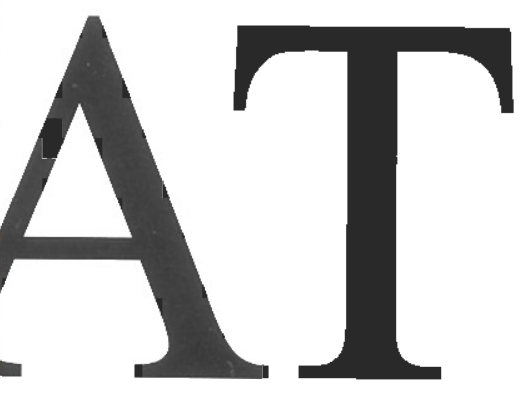

Conferencias, seminarios y trabajos de Matemática

IS SN: $1515-4904$

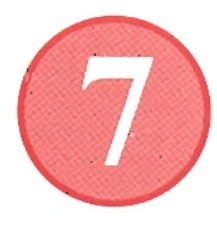

Primeras Jornadas

sobre Ecuaciones

Diferenciales,

Optimización y

Análisis Numérico

Primera Parte 
MAT

\section{SERIE A: CONFERENCIAS, SEMINARIOS Y TRABAJOS DE MATEMÁTICA}

No. 7

\section{PRIMERAS JORNADAS SOBRE ECUACIONES DIFERENCIALES, OPTIMIZACIÓN Y ANÁLISIS NUMÉRICO}

Primera Parte

Domingo A. Tarzia (Ed.)

\section{INDICE}

Adriana B. Verdiell - María C. Maciel - Susana L. Orofino - Tatiana I. Gibelli, "A survey of the spectral gradient method”, 1-14.

María F. Natale - Domingo A. Tarzia, "An integral equation in order to solve a one-phase Stefan problem with nonlinear thermal conductivity”, 15-24.

María C. Sanziel - Domingo A. Tarzia, "Optimization on the heat flux in a mixed elliptic problem with temperature constraints”, 25-30.

Claudia M. Gariboldi - Domingo A. Tarzia, "A new proof of the convergence of distributed optimal controls on the internal energy in mixed elliptic problems”, 3142. 


\title{
A NEW PROOF OF THE CONVERGENCE OF DISTRIBUTED OPTIMAL CONTROLS ON THE INTERNAL ENERGY IN MIXED ELLIPTIC PROBLEMS *
}

\author{
Claudia M. Gariboldi (1) - Domingo A. Tarzia (2)
}

(1) Depto. Matemática, FCEFQyN, Univ. Nac. de Rio Cuarto, Ruta $36 \mathrm{Km}$ 601, 5800 Rio Cuarto, Argentina.

E-mail: cgariboldi@exa.unrc.edu.ar

(2) Depto. Matemática-CONICET, FCE, Univ. Austral, Paraguay 1950, S2000FZF Rosario, Argentina.

E-mail: Domingo.Tarzia@fce.austral.edu.ar

\begin{abstract}
We consider two steady-state heat conduction problems $P$ and $P_{\alpha}$ (for each $\alpha>$ 0 ) with mixed boundary conditions for the same Poisson equation. The difference between both problems is that on the boundary portion $\Gamma_{1}$ a Dirichlet condition is verified for $P$ and a Newton condition with transfer coefficient $\alpha$ is verified for $P_{\alpha}$. We formulate distributed optimal control problems, for a suitable cost function, over the internal energy $g$ in the material. We make a new proof with respect to the one given in C.M. Gariboldi - D.A. Tarzia, App. Math. Optim. 47 (2003), 213-230 on the strongly convergence when $\alpha \rightarrow \infty$ of the optimal control $g_{o p_{\alpha}}$, the system state $u_{g_{o p_{\alpha} \alpha}}$ and the adjoint state $p_{g_{o p_{\alpha} \alpha}}$ to the optimal control $g_{o p}$, system state $u_{g_{o p}}$ and adjoint state $p_{g_{o p}}$ corresponding to $P_{\alpha}$ and $P$ respectively. For this proof we eliminate the restriction on the constant of coerciveness of the bilinear form, and we use properties of the cost function and the theory of variational equalities instead of the fixed point theorem.
\end{abstract}

Key words: Variational Inequality, Distributed Optimal Control, Mixed Elliptic Problem, Adjoint State, Steady-State Stefan Problem, Optimality Condition.

AMS Subject Classifications: 49J20, 35J85, 35R35.

${ }^{*}$ MAT - Serie A, 7 (2004), 31-42. 


\section{Introduction}

We consider a bounded domain $\Omega$ in $\mathbb{R}^{n}$ whose regular boundary $\Gamma$ consists of the union of two disjoint portions $\Gamma_{1}$ y $\Gamma_{2}$ with meas $\left(\Gamma_{1}\right)>0$ and meas $\left(\Gamma_{2}\right)>0$. We denote with meas $(\Gamma)$ the $(n-1)$-dimensional Lebesgue measure of $\Gamma$.

We consider the following two steady-state heat conduction problems $\mathrm{P}$ and $\mathrm{P}_{\alpha}$ (for each parameter $\alpha>0$ ) respectively with mixed boundary conditions:

$$
-\Delta u=g \text { in }\left.\Omega \quad u\right|_{\Gamma_{1}}=B \quad-\left.\frac{\partial u}{\partial n}\right|_{\Gamma_{2}}=q
$$

and

$$
-\Delta u=g \text { in } \Omega \quad-\left.\frac{\partial u}{\partial n}\right|_{\Gamma_{1}}=\alpha(u-B) \quad-\left.\frac{\partial u}{\partial n}\right|_{\Gamma_{2}}=q
$$

where $g$ is the internal energy in $\Omega$, B is the temperature on $\Gamma_{1}$ for $(1)$ and the temperature of the external neighboord of $\Gamma_{1}$ for $(2), q$ is the heat flux on $\Gamma_{2}$ and $\alpha>0$ is the heat transfer coefficient of $\Gamma_{1}$ (Newton's law on $\Gamma_{1}$ ), that satisfy the following assumptions:

$$
g \in H=L^{2}(\Omega), \quad q \in L^{2}\left(\Gamma_{2}\right), \quad B \in H^{\frac{1}{2}}\left(\Gamma_{1}\right) .
$$

Problems (1) and (2) can be considered as the steady-state Stefan problem for suitable data $q, g$ and B [5], [8], [11], [18], [19] and [21] .

Let $u_{g}$ and $u_{g \alpha}$ be the unique solutions of the mixed elliptic problems (1) and (2) respectively whose variational equalities are given by [15], [19]:

$$
a\left(u_{g}, v\right)=L_{g}(v), \forall v \in V_{0}, u_{g} \in K
$$

and

$$
a_{\alpha}\left(u_{g \alpha}, v\right)=L_{g \alpha}(v), \forall v \in V, u_{g \alpha} \in V
$$

where

$$
\begin{gathered}
V=H^{1}(\Omega) ; \quad V_{0}=\left\{v \in V /\left.v\right|_{\Gamma_{1}}=0\right\} \\
K=v_{0}+V_{0} ; \quad(g, h)=(g, h)_{H}=\int_{\Omega} g h d x ; \\
a(u, v)=\int_{\Omega} \nabla u . \nabla v d x ; \quad a_{\alpha}(u, v)=a(u, v)+\alpha \int_{\Gamma_{1}} B v d \gamma \\
L_{g}(v)=(g, v)_{H}-\int_{\Gamma_{2}} q v d \gamma ; \quad L_{g \alpha}(v)=L_{g}(v)+\alpha \int_{\Gamma_{1}} B v d \gamma
\end{gathered}
$$

for a given $v_{0} \in V,\left.v_{0}\right|_{\Gamma_{1}}=B$.

We consider $g$ as a control variable for the cost functionals $J: H \rightarrow \mathbb{R}_{0}^{+}$and $J_{\alpha}$ : $H \rightarrow \mathbb{R}_{0}^{+}$respectively given by: 


$$
J(g)=\frac{1}{2}\left\|u_{g}-z_{d}\right\|_{H}^{2}+\frac{M}{2}\|g\|_{H}^{2}
$$

and

$$
J_{\alpha}(g)=\frac{1}{2}\left\|u_{g \alpha}-z_{d}\right\|_{H}^{2}+\frac{M}{2}\|g\|_{H}^{2}
$$

where $z_{d} \in H$ is given and $M=$ const. $>0$.

Then we can formulate the following distributed optimal control problems [7], [9], [10], $[12]$ and [16]:

$$
\text { Find } g_{o p} \in H \text { such that } J\left(g_{o p}\right)=\min _{g \in H} J(g)
$$

and

$$
\text { Find } g_{o p_{\alpha}} \in H \text { such that } J_{\alpha}\left(g_{o p_{\alpha}}\right)=\min _{g \in H} J_{\alpha}(g)
$$

respectively.

The use of the variational inequality theory in connection with optimal control problems was done, for example, in [1], [2], [3], [4], [6], [14] and [17]. In [13] an optimization problem corresponding to (1) is studied in order to avoid a change phase process.

In Section 2 we get that the functional $J$ is coercive and Gâteaux differentiable on $H$, $J^{\prime}$ is a lipschitzian and strictly monotone application on $H$. We also obtain the existence and uniqueness of the distributed optimal control problem (9). Similary, in Section 3 we get that the functional $J_{\alpha}$ is coercive and Gâteaux differentiable on $H, J_{\alpha}^{\prime}$ is a lipschitzian and strictly monotone application on $H$ for all $\alpha>0$. We also obtain the existence and uniqueness of the distributed optimal control problem (10) and strongly convergence (when $\alpha \rightarrow \infty$ ) of the states system (2) and the corresponding adjoint states to the respectives of the system (1), for all $g \in H$. Sections 2 and 3 follow [12].

In Section 4 we study the convergence when $\alpha \rightarrow \infty$ of the optimal control problem (10) corresponding to the state system (2). We prove that the optimal state system $u_{g_{o p_{\alpha} \alpha}}$ and the optimal adjoint system $p_{g_{o p_{\alpha}} \alpha}$ of problem (10) are strongly convergent in $\mathrm{V}$ to the

corresponding $u_{g_{o p}}$ and $p_{g_{o p}}$ for problem (9) respectively when $\alpha \rightarrow \infty$. Finally the strong convergence in $H$ of the optimal control $g_{o p_{\alpha}}$ of problem (10) to the optimal control $g_{o p}$ of problem (9) is also proved when $\alpha \rightarrow \infty$. This proof is new with respect to the one given in [12]. We have eliminated the restriction on the constant of coerciveness of the bilinear form $a$ and we use the variational equality theory and the optimal control problem instead of the fixed point theory.

\section{Problem $\mathrm{P}$ and its Corresponding Optimal Control Problem}

Let $C: H \rightarrow V_{0}$ be the application such that:

$$
C(g)=u_{g}-u_{0}
$$

where $u_{0}$ is the solution of problem (4) for $g=0$ whose variational equality is given by: 


$$
a\left(u_{0}, v\right)=L_{0}(v), \forall v \in V_{0}, u_{0} \in K
$$

with

$$
L_{0}(v)=-\int_{\Gamma_{2}} q v d \gamma
$$

Let $\Pi: H \times H \rightarrow \mathbb{R}$ and $\mathrm{L}: H \rightarrow \mathbb{R}$ be defined by the following expressions:

$$
\begin{gathered}
\Pi(g, h)=(C(g), C(h))+M(g, h), \quad \forall g, h \in H \\
L(g)=\left(C(g), z_{d}-u_{0}\right), \quad \forall g \in H .
\end{gathered}
$$

We have that $a$ is a bilinear, continuous and symmetric form on $V$ and coercive on $V_{0}$, that is [15], [19]:

$$
\exists \lambda>0 \text { such that } a(v, v) \geq \lambda\|v\|_{V}^{2}, \forall v \in V_{0} .
$$

Lemma 2.1. (i) $C$ is a linear and continuous application, $\Pi$ is a linear, continuous, symmetric and coercive form on $H$, that is:

$$
\Pi(g, g) \geq M\|g\|_{H}^{2}, \forall g \in H
$$

and $L$ is linear and continuous on $H$.

(ii) $J$ can be also written as:

$$
J(g)=\frac{1}{2} \Pi(g, h)-L(g)+\frac{1}{2}\left\|u_{0}-z_{d}\right\|_{H}^{2}, \forall g \in H .
$$

(iii) There exists a unique optimal control $g_{o p} \in H$ such that:

$$
J\left(g_{o p}\right)=\min _{g \in H} J(g)
$$

(iv) The application $g \in H \rightarrow u_{g} \in V$ is lipschitzian, that is:

$$
\left\|u_{g_{2}}-u_{g_{1}}\right\|_{V} \leq \frac{1}{\lambda}\left\|g_{2}-g_{1}\right\|_{H}, \forall g_{1}, g_{2} \in H
$$

(v) $J$ is a Gâteaux differentiable functional and $J^{\prime}$ is given by:

$$
\left\langle J^{\prime}(g), h\right\rangle=\left(u_{g}-z_{d}, C(h)\right)+M(g, h)=\Pi(g, h)-L(g), \quad \forall g, h \in H .
$$

(vi) The Gâteaux derivative of $J$ can be written as:

$$
J^{\prime}(g)=p_{g}+M g, \forall g \in H .
$$

where the adjoint state $p_{g}$ corresponding to problem (1) or (4), for each $g \in H$, is the unique solution of the following mixed elliptic problem:

$$
-\Delta p_{g}=u_{g}-z_{d} \text { in } \Omega ;\left.p_{g}\right|_{\Gamma_{1}}=0 ;\left.\frac{\partial p_{g}}{\partial n}\right|_{\Gamma_{2}}=0
$$

whose variational formulation is given by: 


$$
a\left(p_{g}, v\right)=\left(u_{g}-z_{d}, v\right), \forall v \in V_{0}, p_{g} \in V_{0} .
$$

Moreover, the adjoint state $p_{g}$ satisfy the following equalities:

$$
\left(p_{g}, h\right)=\left(u_{g}-z_{d}, C(h)\right)=a\left(p_{g}, C(h)\right) \forall g, h \in H .
$$

(vii) The optimality condition for the problem (9) is given by $J^{\prime}\left(g_{o p}\right)=0$ in $H$, that is:

$$
p_{g_{o p}}+M g_{o p}=0 \text { in } H
$$

(viii) We have the following inequality:

$$
\left\|p_{g_{2}}-p_{g_{1}}\right\|_{V} \leq \frac{1}{\lambda}\left\|u_{g_{2}}-u_{g_{1}}\right\|_{H} \forall g_{1}, g_{2} \in H
$$

Proof. (i)-(iv) See [12].

(v)-(viii) The mean ideas of the proof are the following expressions:

$$
\begin{array}{r}
\begin{aligned}
a) \frac{1}{t}[J(g+t(f-g))-J(g)]= & \frac{t}{2}\left(u_{f}-u_{g}, u_{f}-u_{g}\right)+\left(u_{g}-z_{d}, u_{f}-u_{g}\right) \\
& +M(g, f-g)+\frac{M t}{2}(f-g, f-g)
\end{aligned} \\
\begin{aligned}
\text { b) } a\left(p_{g}, C(h)\right)=a\left(p_{g}, u_{h}-u_{0}\right)=a\left(p_{g}, u_{h}\right)-a\left(p_{g}, u_{0}\right)=\left(p_{g}, h\right) \\
\text { c) } \lambda\left\|p_{g_{2}}-p_{g_{1}}\right\|_{V}^{2} \leq a\left(p_{g_{2}}-p_{g_{1}}, p_{g_{2}}-p_{g_{1}}\right) \leq\left\|u_{g_{2}}-u_{g_{1}}\right\|_{H}\left\|p_{g_{2}}-p_{g_{1}}\right\|_{H}
\end{aligned}
\end{array}
$$

and the details are given in [12].

Now, we are in conditions for obtaining other properties of the functional $J$.

Lemma 2.2. (i) The application $g \in H \rightarrow p_{g} \in V_{0}$ is strictly monotone. Moreover, we have:

$$
\left(p_{g_{2}}-p_{g_{1}}, g_{2}-g_{1}\right)=\left\|u_{g_{2}}-u_{g_{1}}\right\|_{H}^{2} \geq 0, \quad \forall g_{1}, g_{2} \in H .
$$

(ii) $J$ is coercive or $H$-elliptic, that is:

$$
(1-t) J\left(g_{2}\right)+t J\left(g_{1}\right)-J\left((1-t) g_{2}+t g_{1}\right) \geq \frac{M t(1-t)}{2}\left\|g_{2}-g_{1}\right\|_{H}^{2}, \quad \forall g_{1}, g_{2} \in H, \quad \forall t \in[0,1] .
$$

(iii) $J^{\prime}$ is a Lipschitzian and strictly monotone application, that is:

$$
\left\|J^{\prime}\left(g_{2}\right)-J^{\prime}\left(g_{1}\right)\right\|_{H} \leq\left(M+\frac{1}{\lambda^{2}}\right)\left\|g_{1}-g_{2}\right\|_{H}
$$

and

$$
\left\langle J^{\prime}\left(g_{2}\right)-J^{\prime}\left(g_{1}\right), g_{2}-g_{1}\right\rangle=\left\|u_{g_{2}}-u_{g_{1}}\right\|_{H}^{2}+M\left\|g_{2}-g_{1}\right\|_{H}^{2} \geq M\left\|g_{2}-g_{1}\right\|_{H}^{2}, \quad \forall g_{1}, g_{2} \in H .
$$




\section{Problem $\mathbf{P}_{\alpha}$ and Its Corresponding Optimal Con- trol Problem}

Let $\Pi_{\alpha}: H \times H \rightarrow \mathbb{R}, L_{\alpha}: H \rightarrow \mathbb{R}$ and $C_{\alpha}: H \rightarrow V$ be defined by:

$$
\begin{gathered}
\Pi_{\alpha}(g, h)=\left(C_{\alpha}(g), C_{\alpha}(h)\right)+M(g, h), \forall g, h \in H \\
L_{\alpha}(g)=\left(C_{\alpha}(g), z_{d}-u_{0 \alpha}\right), \quad \forall g \in H \\
C_{\alpha}(g)=u_{g \alpha}-u_{0 \alpha}, \quad \forall g \in H
\end{gathered}
$$

where $u_{g \alpha}$ is the unique solution of the variational equality (5), $u_{0 \alpha}$ is the unique solution of (5) for $g=0$ whose variational equality is given by:

$$
a_{\alpha}\left(u_{0 \alpha}, v\right)=L_{0 \alpha}(v), \forall v \in V, u_{0 \alpha} \in V
$$

with

$$
L_{0 \alpha}(v)=\alpha \int_{\Gamma_{1}} B v d \gamma-\int_{\Gamma_{2}} q v d \gamma
$$

and $a_{\alpha}$ is a bilinear, continuous, symmetric and coercive form on $V$, that is:

$$
a_{\alpha}(v, v) \geq \lambda_{\alpha}\|v\|_{V}^{2}, \forall v \in V .
$$

where $\lambda_{\alpha}=\lambda_{1} \min (1, \alpha)>0$ for all $\alpha>0$ and $\lambda_{1}$ is the coerciveness constant for the bilinear form $a_{1}[20]$.

We can obtain similaries properties to Lemma 2.1, following [13], [15], [16] and [19] which proof is omitted.

Lemma 3.1. Let $\alpha>0$ be. (i) $C_{\alpha}$ is a linear and continuous application, $\Pi_{\alpha}$ is linear, continuous, symmetric and coercive on $H$, that is:

$$
\Pi_{\alpha}(g, g) \geq M\|g\|_{H}^{2}, \forall g \in H .
$$

and $L_{\alpha}$ is linear and continuous on $H$.

(ii) $J_{\alpha}$ can be also written as:

$$
J_{\alpha}(g)=\frac{1}{2} \Pi_{\alpha}(g, h)-L_{\alpha}(g)+\frac{1}{2}\left\|u_{0 \alpha}-z_{d}\right\|_{H}^{2}, \forall g \in H .
$$

(iii) There exists a unique optimal control $g_{o p_{\alpha}} \in H$ such that:

$$
J_{\alpha}\left(g_{o p_{\alpha}}\right)=\min _{g \in H} J_{\alpha}(g) .
$$

(iv) The application $g \in H \rightarrow u_{g \alpha} \in V$ is lipschitzian, that is:

$$
\left\|u_{g_{2} \alpha}-u_{g_{1} \alpha}\right\|_{V} \leq \frac{1}{\lambda_{\alpha}}\left\|g_{2}-g_{1}\right\|_{H}, \forall g_{1}, g_{2} \in H .
$$

(v) $J_{\alpha}$ is Gâteaux differentiable functional and $J_{\alpha}^{\prime}$ is given by: 
Gariboldi-Tarzia, "Convergence controls ...", MAT-Serie A, 7 (2004), 31-42

$$
\left\langle J_{\alpha}^{\prime}(g), h\right\rangle=\left(u_{g \alpha}-z_{d}, C_{\alpha}(h)\right)+M(g, h)=\Pi_{\alpha}(g, h)-L_{\alpha}(g), \forall g, h \in H
$$

(vi) The Gâteaux derivative of $J_{\alpha}$ can be written as:

$$
J_{\alpha}^{\prime}(g)=p_{g \alpha}+M g, \forall g \in H
$$

where the adjoint state $p_{g \alpha}$ is the unique solution of the following mixed elliptic problem corresponding to (2) or (5), for each $g \in H$ and $\alpha>0$ :

$$
-\Delta p_{g \alpha}=u_{g \alpha}-z_{d} \text { in } \Omega ;-\left.\frac{\partial p_{g \alpha}}{\partial n}\right|_{\Gamma_{1}}=\alpha p_{g \alpha} ;\left.\frac{\partial p_{g \alpha}}{\partial n}\right|_{\Gamma_{2}}=0
$$

whose variational formulation is given by:

$$
a_{\alpha}\left(p_{g \alpha}, v\right)=\left(u_{g \alpha}-z_{d}, v\right), \forall v \in V, p_{g \alpha} \in V .
$$

where $u_{g \alpha}$ is the unique solution of (5). Moreover, the adjoint state $p_{g \alpha}$ satisfies the following equalities:

$$
\left(p_{g \alpha}, h\right)=\left(u_{g \alpha}-z_{\alpha}, C_{\alpha}(h)\right)=a_{\alpha}\left(p_{g \alpha}, C_{\alpha}(h)\right), \forall g, h \in H .
$$

(vii) The optimality condition for problem (10) is given by $J_{\alpha}^{\prime}\left(g_{o p_{\alpha}}\right)=0$ in $H$, that is:

$$
p_{g_{o p_{\alpha} \alpha}}+M g_{o p_{\alpha}}=0 \text { in } H \text {. }
$$

(viii) We have the following property:

$$
\left\|p_{g_{2 \alpha}}-p_{g_{1 \alpha}}\right\|_{V} \leq \frac{1}{\lambda_{\alpha}}\left\|u_{g_{2 \alpha}}-u_{g_{1 \alpha}}\right\|_{H} \forall g_{1 \alpha}, g_{2 \alpha} \in H
$$

Proof. See [12]

Remark 1. We note the double dependence on the parameter $\alpha$ for the optimal state system $u_{g_{o p_{\alpha} \alpha} \alpha}$ and the adjoint state $p_{g_{o p_{\alpha}} \alpha}$.

Lemma 3.2. (i) The operator $g \in H \rightarrow p_{g \alpha} \in V$ is strictly monotone, that is:

$$
\left(p_{g_{2} \alpha}-p_{g_{1} \alpha}, g_{2}-g_{1}\right)=\left\|u_{g_{2} \alpha}-u_{g_{1} \alpha}\right\|_{H}^{2} \geq 0, \forall g_{1}, g_{2} \in H .
$$

(ii) $J_{\alpha}$ is coercive or $H$-elliptic, that is:

$$
(1-t) J_{\alpha}\left(g_{2}\right)+t J_{\alpha}\left(g_{1}\right)-J_{\alpha}\left((1-t) g_{2}+t g_{1}\right) \geq \frac{M t(1-t)}{2}\left\|g_{2}-g_{1}\right\| a_{H}^{2}, \forall g_{1}, g_{2} \in H ; \forall t \in[0,1] .
$$

(iii) $J_{\alpha}^{\prime}$ is a Lipschitzian and strictly monotone operator, that is:

$$
\left\|J_{\alpha}^{\prime}\left(g_{2}\right)-J_{\alpha}^{\prime}\left(g_{1}\right)\right\|_{H} \leq\left(M+\frac{1}{\lambda_{\alpha}^{2}}\right)\left\|g_{1}-g_{2}\right\|_{H}, \forall g_{1}, g_{2} \in H
$$

and

$$
\left\langle J_{\alpha}^{\prime}\left(g_{2}\right)-J_{\alpha}^{\prime}\left(g_{1}\right), g_{2}-g_{1}\right\rangle \geq M\left\|g_{2}-g_{1}\right\|_{H}^{2}, \forall g_{1}, g_{2} \in H .
$$

Proof. See $[12]$

Now, we will prove the following result of convergence when $\alpha \rightarrow \infty$. 
Lemma 3.3. For all $\alpha>0, q \in L^{2}\left(\Gamma_{2}\right), B \in H^{\frac{1}{2}}\left(\Gamma_{1}\right)$, we have the following limits:

$$
\begin{aligned}
\text { i) } \lim _{\alpha \rightarrow \infty}\left\|u_{g \alpha}-u_{g}\right\|_{V} & =0, \forall g \in H \\
\text { ii) } \lim _{\alpha \rightarrow \infty}\left\|u_{0 \alpha}-u_{0}\right\|_{V} & =0 \\
\text { iii) } \lim _{\alpha \rightarrow \infty}\left\|p_{g \alpha}-p_{g}\right\|_{V} & =0, \forall g \in H .
\end{aligned}
$$

Proof. (i) Taking into account [12] and following [19] and [20] we obtain that there exists $C_{1}$, a constant independient of $\alpha$, such that for large $\alpha$ :

$$
\left\|u_{g \alpha}-u_{g}\right\|_{V}^{2} \leq \frac{C_{1}}{\lambda_{1}} \quad, \quad(\alpha-1) \int_{\Gamma_{1}}\left(u_{g \alpha}-u_{g}\right)^{2} d \gamma \leq \frac{\left(C_{1}\right)^{2}}{\lambda_{1}}
$$

and we deduce that there exists $w_{g} \in K$ such that:

$$
a\left(w_{g}, v\right)=L_{g}(v), \forall v \in V_{0}, w_{g} \in K
$$

and by uniqueness, we have $w_{g}=u_{g}$. Therefore, $u_{g \alpha} \rightarrow u_{g}$ strongly in $V$ as $\alpha \rightarrow \infty$ because the following inequality:

$$
\lambda_{1}\left\|u_{g \alpha}-u_{g}\right\|_{V}^{2} \leq L_{g}\left(u_{g \alpha}-u_{g}\right)-a\left(u_{g \alpha}, u_{g \alpha}-u_{g}\right) .
$$

For the case (ii) we take $g=0$ in the case (i).

(iii) We prove that there exists $C_{2}$ a constant independent of $\alpha$, for large $\alpha$, such that:

$$
\left\|p_{g \alpha}-p_{g}\right\|_{V}^{2} \leq \frac{C_{2}}{\lambda_{1}} \quad, \quad(\alpha-1) \int_{\Gamma_{1}}\left(p_{g \alpha}-p_{g}\right)^{2} d \gamma \leq \frac{\left(C_{2}\right)^{2}}{\lambda_{1}}
$$

and we deduce that there exists $\xi_{g} \in V_{0}$ such that:

$$
a\left(\xi_{g}, v\right)=\left(u_{g}-z_{d}, v\right), \forall v \in V_{0}, \xi_{g} \in V_{0} .
$$

and by uniqueness, we obtain $\xi_{g}=p_{g}$. Therefore, taking into account the following inequality:

$$
\lambda_{1}\left\|p_{g \alpha}-p_{g}\right\|_{V}^{2} \leq\left(u_{g \alpha}-z_{d}, p_{g \alpha}-p_{g}\right)-a\left(p_{g}, p_{g \alpha}-p_{g}\right)
$$

we get that $p_{g \alpha} \rightarrow p_{g}$ strongly in $V$.

\section{Convergence of Problem $\mathrm{P}_{\alpha}$ and its Corresponding Optimal Control as $\alpha \rightarrow \infty$}

In this section we will make a new proof with respect to the one given in [12] of the strongly convergence of the optimal control $g_{o p_{\alpha}}$ of problem (10) and its corresponding adjoint state $p_{g_{o p_{\alpha} \alpha}}(41)$ to the optimal control $g_{o p}$ of problem (9) and its corresponding adjoint state $p_{g_{o p}}(22)$ respectively when the parameter $\alpha$ (heat transfer coefficient on $\Gamma_{1}$ ) goes to infinity. We will eliminate the restriction on the constant of coerciveness of the bilinear form $a$ and we will use properties of the cost function and the variational equality theory instead of the fixed point theorem. 
Gariboldi-Tarzia, "Convergence controls ...", MAT-Serie A, 7 (2004), 31-42

Theorem 4.1. (i) If $p_{g_{o p}}$ and $p_{g_{o p_{\alpha} \alpha}}$ are the corresponding adjoint state of the problem (9) and problem (10) respectively, then:

$$
\lim _{\alpha \rightarrow \infty}\left\|p_{g_{o p_{\alpha} \alpha}}-p_{g_{o p}}\right\|_{V}=0
$$

(ii) If $g_{o p}$ and $g_{o p_{\alpha}}$ are the solutions of the problem (9) and problem (10) respectively, then:

$$
\lim _{\alpha \rightarrow \infty}\left\|g_{o p_{\alpha}}-g_{o p}\right\|_{H}=0 .
$$

(iii) If $u_{g_{o p}}$ and $u_{g_{o p_{\alpha} \alpha}}$ are the corresponding solutions of the problem $\mathrm{P}$ and problem $\mathrm{P}_{\alpha}$ respectively, then:

$$
\lim _{\alpha \rightarrow \infty}\left\|u_{g_{o p_{\alpha} \alpha}}-u_{g_{o p}}\right\|_{V}=0 .
$$

Proof. First we will prove some preliminary results. Since $g_{o p_{\alpha}}$ is the solution of the problem (10), we have the following inequality:

$$
\frac{1}{2}\left\|u_{g_{o p_{\alpha} \alpha} \alpha}-z_{d}\right\|_{H}^{2}+\frac{M}{2}\left\|g_{o p_{\alpha}}\right\|_{H}^{2} \leq \frac{1}{2}\left\|u_{g \alpha}-z_{d}\right\|_{H}^{2}+\frac{M}{2}\|g\|_{H}^{2}, \forall g \in H,
$$

then, taking $g=0$, we have:

$$
\frac{1}{2}\left\|u_{g_{o p_{\alpha} \alpha}}-z_{d}\right\|_{H}^{2}+\frac{M}{2}\left\|g_{o p_{\alpha}}\right\|_{H}^{2} \leq \frac{1}{2}\left\|u_{0 \alpha}-z_{d}\right\|_{H}^{2} \leq C_{3}, \forall \alpha>0,
$$

where $C_{3}$ is a constant independent of parameter $\alpha$ because $u_{0 \alpha}$ is convergent when $\alpha \rightarrow \infty$. Therefore

$$
\left\|g_{o p_{\alpha}}\right\|_{H} \leq C_{4} \text { and }\left\|u_{g_{o p_{\alpha} \alpha} \alpha}\right\|_{H} \leq C_{5}
$$

where $C_{4}$ and $C_{5}$ are constants independent of $\alpha$. Now, if we take $v=u_{g_{o p_{\alpha} \alpha}}-u_{g_{o p}}$ in the variational equality (5), following [19] and [20] we obtain for $\alpha>1$ :

$$
\begin{aligned}
\lambda_{1}\left\|u_{g_{o p} \alpha}-u_{g_{o p}}\right\|_{V}^{2}+(\alpha-1) \int_{\Gamma_{1}}\left(u_{g_{o p} \alpha}-u_{g_{o p}}\right)^{2} d \gamma & \leq a_{\alpha}\left(u_{g_{o p_{\alpha} \alpha}}-u_{g_{o p}}, u_{g_{o p} \alpha}-u_{g_{o p}}\right) \\
& \leq C_{6}\left\|u_{g_{o p_{\alpha} \alpha} \alpha}-u_{g_{o p}}\right\|_{V}
\end{aligned}
$$

where $C_{6}=C_{6}\left(g_{o p}, q, u_{g_{o p}}\right)$ is independent of $\alpha$. Next, we have:

$$
\left\|u_{g_{o p_{\alpha} \alpha} \alpha}-u_{g_{o p}}\right\|_{V}^{2} \leq \frac{C_{6}}{\lambda_{1}} \quad, \quad(\alpha-1) \int_{\Gamma_{1}}\left(u_{g_{o p_{\alpha} \alpha} \alpha}-u_{g_{o p}}\right)^{2} d \gamma \leq \frac{\left(C_{6}\right)^{2}}{\lambda_{1}}
$$

and therefore we deduce that:

$$
\exists \eta \in V \text { sucht that } u_{g_{o p_{\alpha} \alpha}} \rightarrow \eta \text { weakly in } V,
$$

and because the following inequalities:

$$
0 \leq \int_{\Gamma_{1}}\left(\eta-u_{g_{o p}}\right)^{2} d \gamma \leq \lim _{\alpha \rightarrow \infty} \inf _{\Gamma_{1}}\left(u_{g_{o p_{\alpha} \alpha}}-u_{g_{o p}}\right)^{2} d \gamma=0,
$$


we obtain that $\eta \in K$. Next, if we take $v=p_{g_{o p_{\alpha} \alpha}}-p_{g_{o p}}$ in the variational equality (41) we get:

$$
\begin{aligned}
\lambda_{1}\left\|p_{g_{o p_{\alpha} \alpha} \alpha}-p_{g_{o p}}\right\|_{V}^{2}+(\alpha-1) \int_{\Gamma_{1}}\left(p_{g_{o p_{\alpha} \alpha} \alpha}-p_{g_{o p}}\right)^{2} d \gamma & \leq a_{\alpha}\left(p_{g_{o p_{\alpha} \alpha} \alpha}-p_{g_{o p}}, p_{g_{o p_{\alpha}} \alpha}-p_{g_{o p}}\right) \\
& \leq C_{7}\left\|p_{g_{o p_{\alpha}} \alpha}-p_{g_{o p}}\right\|_{V}
\end{aligned}
$$

with $C_{7}=C_{7}\left(C_{5}, p_{g_{o p}}\right)$. Next, we obtain:

$$
\left\|p_{g_{o p_{\alpha} \alpha} \alpha}-p_{g_{o p}}\right\|_{V}^{2} \leq \frac{C_{7}}{\lambda_{1}} \quad, \quad(\alpha-1) \int_{\Gamma_{1}}\left(p_{g_{o p_{\alpha}} \alpha}-p_{g_{o p}}\right)^{2} d \gamma \leq \frac{\left(C_{7}\right)^{2}}{\lambda_{1}}
$$

and therefore we deduce that:

$$
\exists \xi \in V \text { such that } p_{g_{o p_{\alpha} \alpha}} \rightarrow \xi \text { weakly in } V
$$

and by the following inequality:

$$
0 \leq \int_{\Gamma_{1}}\left(\xi-p_{g_{o p}}\right)^{2} d \gamma \leq \lim _{\alpha \rightarrow \infty} \inf _{\Gamma_{1}}\left(p_{g_{o p_{\alpha}} \alpha}-p_{g_{o p}}\right)^{2} d \gamma=0
$$

we obtain $\xi \in V_{0}$. Now, we consider $v \in V_{0}$ and taking into acount (59) and (61), from the variational equality (41) we have:

$$
a(\xi, v)=\left(\eta-z_{d}, v\right), \forall v \in V_{0}, \xi \in V_{0} .
$$

Next, from (57) we deduce that there exists $f \in H$ such that $g_{o_{\alpha}} \rightarrow f$ weakly in $H$. Therefore if we put $v \in V_{0}$ in the variational equality (5) and we pass to the limit $\alpha \rightarrow \infty$, we obtain:

$$
a(\eta, v)=(f, v)-\int_{\Gamma_{2}} q v d \gamma, \forall v \in V_{0}, \eta \in K
$$

Now,

$$
a(\eta, v)=L_{f}(v), \forall v \in V_{0}, \eta \in K
$$

and from the uniqueness of solution of the variational equality (4), we have:

$$
\eta=u_{f}
$$

On the oder hand, from (62), (65) and the uniqueness of solution of the variational equality (22), it results that:

$$
\xi=p_{f}
$$

Now,

$$
J_{\alpha}\left(g_{o p_{\alpha}}\right) \leq J_{\alpha}\left(f^{*}\right), \forall f^{*} \in H
$$

next,

$$
J(f)=J_{\alpha}(f) \leq \lim _{\alpha \rightarrow \infty} \inf J_{\alpha}\left(g_{o p_{\alpha}}\right) \leq \lim _{\alpha \rightarrow \infty} \inf _{\alpha} J_{\alpha}\left(f^{*}\right)=\lim _{\alpha \rightarrow \infty} J_{\alpha}\left(f^{*}\right)=J\left(f^{*}\right)
$$


Gariboldi-Tarzia, "Convergence controls ...", MAT-Serie A, 7 (2004), 31-42

and from the uniqueness of the optimal control we obtain that $f=g_{o p}$. Therefore $\eta=$ $u_{f}=u_{g_{o p}}$ and $\xi=p_{f}=p_{g_{o p}}$.

Moreover, from (61) and the following computation:

$$
\begin{aligned}
\lambda_{1}\left\|p_{g_{o p_{\alpha} \alpha} \alpha}-p_{g_{o p}}\right\|_{V}^{2} & \leq a_{\alpha}\left(p_{g_{o p_{\alpha} \alpha} \alpha}-p_{g_{o p}}, p_{g_{o p_{\alpha} \alpha} \alpha}-p_{g_{o p}}\right) \\
& =a_{\alpha}\left(p_{g_{o p_{\alpha}} \alpha}, p_{g_{o p_{\alpha} \alpha}}-p_{g_{o p}}\right)-a\left(p_{g_{o p}}, p_{g_{o p_{\alpha} \alpha} \alpha}-p_{g_{o p}}\right) \\
& =\left(u_{g_{o p_{\alpha} \alpha}}-z_{d}, p_{g_{o p_{\alpha}} \alpha}-p_{g_{o p}}\right)-a\left(p_{g_{o p}}, p_{g_{o p_{\alpha} \alpha} \alpha}-p_{g_{o p}}\right)
\end{aligned}
$$

we have (54). From the optimality condition (24) it results that:

$$
\left\|g_{o p_{\alpha}}-g_{o p}\right\|_{H}=\frac{1}{M}\left\|p_{g_{o p}}-p_{g_{o p_{\alpha} \alpha} \alpha}\right\|_{H} \leq \frac{1}{M}\left\|p_{g_{o p}}-p_{g_{o p_{\alpha}} \alpha}\right\|_{V}
$$

and therefore (55) holds. Now, we have:

$$
\begin{aligned}
\lambda_{1}\left\|u_{g_{o p_{\alpha} \alpha} \alpha}-u_{g_{o p}}\right\|_{V}^{2} & \leq a_{\alpha}\left(u_{g_{o p_{\alpha} \alpha}}-u_{g_{o p}}, u_{g_{o p \alpha} \alpha}-u_{g_{o p}}\right) \\
& =a_{\alpha}\left(u_{g_{o p_{\alpha} \alpha} \alpha}, u_{g_{o p_{\alpha} \alpha} \alpha}-u_{g_{o p}}\right)-a_{\alpha}\left(u_{g_{o p}}, u_{g_{o p} \alpha}-u_{g_{o p}}\right) \\
& =L_{g_{o p_{\alpha} \alpha}}\left(u_{g_{o p_{\alpha} \alpha} \alpha}-u_{g_{o p}}\right)-a\left(u_{g_{o p}}, u_{g_{o p_{\alpha} \alpha} \alpha}-u_{g_{o p}}\right)-\alpha \int_{\Gamma_{1}} b\left(u_{g_{o p_{\alpha} \alpha}}-b\right) d \gamma \\
& =a\left(u_{g_{o p_{\alpha}}}, u_{g_{o p_{\alpha} \alpha} \alpha}-u_{g_{o p}}\right)-a\left(u_{g_{o p}}, u_{g_{o p_{\alpha} \alpha} \alpha}-u_{g_{o p}}\right) \\
& =a\left(u_{g_{o p_{\alpha}}}-u_{g_{o p}}, u_{g_{o p_{\alpha} \alpha}}-u_{g_{o p}}\right)
\end{aligned}
$$

and taking into account (55) and the fact that $u_{g_{o p_{\alpha}}} \rightarrow u_{g_{o p}}$ strongly in $V$ when $\alpha \rightarrow \infty$ because (18), we get (56).

\section{Acknowledgements}

This paper has been partially sponsored by the project PIP \# 2000/2000 from CONICETUA, Rosario (Argentina), proyect \# 13900/4 from Fundación Antorchas (Argentina) and ANPCYT PICT \#03-11165 from Agencia (Argentina).

\section{References}

[1] ABERGEL F., "A non-well-posed problem in convex optimal control", Appl. Math. and Optim. 17 (1998), 133-175.

[2] ADAMS D.R. - LENHART S.M. - YONG J., "Optimal control of the obstacle for an elliptic variational inequality", Appl. Math. Optim., 38 (1998), 121-140.

[3] BENSOUSSAN A, "Teoría moderna de control óptimo", Cuadern. Inst. Mat. Beppo Levi, \# 7 (1974).

[4] BERGOUNIUX M., "Optimal control of an obstacle problem", Appl. Math. Optim., 36 (1997), 147-172.

[5] BERRONE L.R. - GARGUICHEVICH G.G., "On a steady Stefan problem for the Poisson equation with flux and Fourier's type boundary conditions", Math. Notae, 36 (1992),49-61. 
[6] CASAS E., "Control of an elliptic problem with pointwise state constraints", SIAM J. Control Optim., 24 (1986), 1309-1318.

[7] CEA J., "Optimisation: théorie et algoritmes", Dunod, Paris (1971).

[8] DUVAUT G., "Problèmes à frontière libre en théorie des milieux continus", Rapport de Recherche \# 185, LABORIA-IRIA, Rocquencourt (1976).

[9] EKELAND I.. - TEMAN R., "Analyse convexe et problèmes variationnelles", DunodGauthier Villars, Paris (1973).

[10] FAURRE P., "Analyse numérique. Notes d'optimization", Ellipses, Paris (1988).

[11] GARGUICHEVICH G.G. - TARZIA D.A., "The steady-state two-phase Stefan problem with an internal energy and some related problems", Atti Sem. Mat. Fís. Univ. Modena, 39 (1991), 615-634.

[12] GARIBOLDI C.M. - TARZIA D.A., "Convergence of distributed optimal controls on the internal energy in mixed elliptic problem when the heat transfer coefficient goes to infinity", Appl. Math. Optim., 47 (2003), 213-230.

[13] GONZALEZ R.L.V. - TARZIA D.A., "Optimization of heat flux in a domain with temperature constraints", J. Optim. Th. Appl., 65 (1990), 245-256.

[14] HASLINGER J. - ROUBİCEK T., "Optimal control of variational inequalities. Aproximation Theory and Numerical Realization", Appl. Math. Optim., 14 (1986), $187-201$.

[15] KINDERLEHRER D. - STAMPACCHIA G., "An introduction to variational inequalities and their applications", Academic Press, New York (1980).

[16] LIONS J.L., " Côntrole optimal des systemes gouvernés par des équations aux dérivées partielles", Dunod-Gauthier Villars, Paris (1968).

[17] MIGNOT F. - PUEL J.P." Optimal control in some variational inequalities", SIAM J. Control Optim., 22 (1984), 466-476.

[18] TABACMAN E.D. - TARZIA D.A., "Sufficient and/or necessary condition for the heat transfer coefficient on $\Gamma_{1}$ and the heat flux on $\Gamma_{2}$ to obtain a steady-state twophase Stefan problem", J. Diff. Eq., 77 (1989), 16-37.

[19] TARZIA D.A., "Sur le problème de Stefan à deux phases", C.R.Acad. Sc. Paris, 288A (1979), 941-944. See also "Etude de l'inéquation variationnelle proposée par Duvaut pour le problème de Stefan à deux phases, II", Bollettino dell'Unione Matematica Italiana, 2B (1983), 589-603.

[20] TARZIA D.A., "Una familia de problemas que converge hacia el caso estacionario del problema de Stefan a dos fases", Math. Notae, 27 (1979), 157-165.

[21] TARZIA D.A., "An inequality for the constant heat flux to obtain a steady-state two-phase Stefan problem", Eng. Anal., 5 (1988), 177-181. 